\section{Concurrent variation of response bias and sensitivity in an operant-psychophysical test}

\author{
MICHAEL TERMAN and JIUAN S. TERMAN* \\ Northeastern University, Boston, Massachusetts 02115
}

The yes-no signal detection procedure was applied to a single-response operant paradigm in which rats discriminated between a standard auditory intensity and attenuated comparison values. The payoff matrix was symmetrical (with reinforcing brain stimulation for correct detections and brief time-out for errors), but signal probability and intensity differences were varied to generate a family of isobias and isosensitivity functions. The d' parameter remained fairly constant across a wide range of bias levels. Isobias functions deviated from a strict matching strategy as discrimination difficulty increased, although an orderly relation was maintained between signal probability value and the degree and direction of response bias.

Psychophysical test procedures converge on operant stimulus control procedures when the contingencies of the psychophysical response are made explicit. Signal detection theory (Green \& Swets, 1966) suggests that the arrangement of reinforcement contingencies can profoundly affect the observer's report of sensory stimulus differences. This suggestive bridge to behavioral formulations of psychophysical judgment (cf. Blough, 1967; Nevin, 1970; Terman, 1970) requires evaluating the relevance of the theory's bias and sensitivity concepts to the analysis of the stimulus control of operant behavior.

Most studies in animal psychophysics have not specified how response bias may interact with sensory variables, and factors such as position preferences have been left to the idiosyncracy of the individual S. A signal detection analysis may help to partial out the potentially confounded bias and sensitivity variables. The degree of bias may be controlled precisely by reinforcement (payoff) and signal probability (expectation) manipulations. The present experiment examined the covariation of response bias and auditory sensitivity in an operant analog to the yes-no signal detection experiment. The design permitted construction of both isosensitivity and isobias functions, affording a sensory analysis in terms of the $d^{\prime}$ parameter of signal detection theory. If observed isosensitivity functions for the rat resembled their theoretical counterparts, it was thought then that the d' parameter could be adopted with confidence in other auditory

*Supported by PHS Grant MH-17892-01 and NASA Grant NGR-22-011-070. Reprints may be obtained from the authors at the Department of Psychology, 440 United Building, Northeastern University, Boston, Massachusetts 02115. experiments, where biasing factors are not explicitly varied.

\section{METHOD \\ Subjects}

Two adult male albino rats of the Charles River CD strain were implanted with bipolar stainless steel electrodes (Plastic Products, Model MS303) in the region of the posterior hypothalamus. They were fed $20 \mathrm{~g}$ of Purina Lab Chow each day and given unlimited access to water in their home cages.

\section{Apparatus}

The psychophysical test chamber was constructed of aluminum front and rear walls with Plexiglas side walls, and measured $0.33 \times 0.24 \times 0.49 \mathrm{~m}$. A Lehigh Valley mouse lever was mounted at the midline of the rear $(0.24 \mathrm{~m})$ wall at the level of the rat's head, with a stimulus setup light (25-mm lens) mounted above it. A Gerbrands pigeon key (which could be "nosed" by the rat) was mounted at the midline of the front wall and was briefly transilluminated following tone presentations (see Procedure).

The chamber was placed inside a ventilated sound-attenuating compartment, with a mercury commutator system for delivery of brain stimulations (Berkley \& Kling, 1967) mounted on the ceiling. Brain stimulations were 0.5 -sec trains of $60-\mathrm{Hz}$ constant-current sine waves, adjusted to an amplitude that maintained rapid responding for each S.

The auditory stimuli were generated by a sine wave oscillator set at $3.0 \mathrm{kHz}$. The oscillator output was cut by two decibel attenuators (to produce standard and comparison intensities), and the sound stimuli were presented for $0.5 \mathrm{sec}$ by an electronic switch. A loudspeaker was suspended from the ceiling of the sound-attenuating compartment, above the rear of the test chamber. Auditory stimulus intensity was calibrated (in decibels re $10^{-4}$ microbar) at the level of the rat's head beneath the speaker, using a sound level meter.

Experimental contingencies were controlled by a hybrid circuit of relays and solid-state programming modules. Standard probability was determined by a Scientific Prototype 4020J probability generator. Data were collected on electromagnetic impulse counters and an event recorder.

\section{Procedure}

The rats were first trained to present and to discriminate successive standard and comparison tones $(p=0.5)$ at $25 \mathrm{~dB}$ difference, according to a procedure for shaping behavior chains (Keller \& Schoenfeld, 1950). In the presence of the stimulus setup light above the bar, a barpress produced a $0.5 \mathrm{sec}$ tone presentation and a 3-sec choice interval, during which the keylight was transilluminated and the setup light was off. If the standard were presented, a keypress occurring during the choice interval would produce a brain-stimulation reinforcer. If the comparison were presented, the reinforcer would be produced at the end of the 3-sec choice interval, providing no keypress was emitted. Pressing the key following comparison presentation produced a 3-sec time-out (during which all experimental contingencies were suspended). Failure to press the key in $3 \mathrm{sec}$ following standard presentation also produced a time-out. The stimulus setup light was presented after each reinforcement or time-out.

In relating these contingencies to a psychophysical framework, the keypress is considered the "yes" report and failure to press the key in the 3-sec choice interval is considered the "no" report. "Yes" following the standard is defined as a hit; "yes" following the comparison is defined as a false alarm; "no" following the comparison is defined as a correct rejection; and "no" following the standard is defined as a miss. The payoff matrix is symmetrical in that hits and correct rejections both produce the reinforcer, while false alarms and misses both produce the time-out.

Following acquisition of errorless response patterns at 25-dB comparison at tenuation, at $p=0.5$, the psychometric test procedure was begun. Each daily session consisted of the ordered presentation of nine comparison intensities with the standard held constant at $100 \mathrm{~dB}$. Following a 350-trial warm-up period 


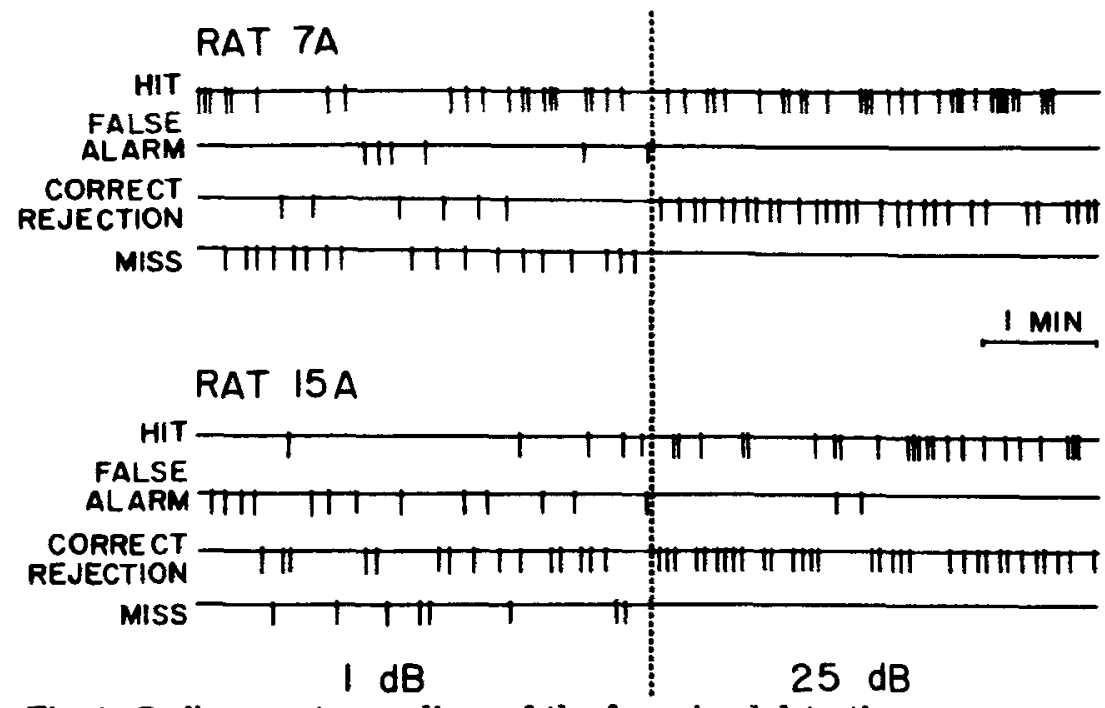

Fig. 1. On-line event recordings of the four signal detection outcomes, taken toward the end of sessions when auditory intensity difference was increased from 1 to $25 \mathrm{~dB}$.

at a 25-dB intensity difference [with $\mathrm{p}(\mathrm{STD})=0.5]$, successive 350 -trial blocks were run at the following comparison attenuation values: 25,20 , $16,13,10,7,5,3$, and $1 \mathrm{~dB}$. Standard probability was varied from session to session in irregular orders, using the following values: $0.1,0.3,0.5,0.7$, and 0.9 . The series of probability values was exhausted before any value was repeated, and each probability value was presented for four sessions. [Sessions at $p($ STD $)=0.4$ were added later for Rat 7A to provide a more complete collection of isobias functions.] In all, 1,400 yes-no reports were collected for each intensity difference at each probability.

While the procedure of decreasing stimulus differences within each session confounds tone intensity with potential temporal effects (fatigue or adaptation?), such a procedure maintains higher levels of differential behavior and thus a finer threshold estimate than random orders or increasing stimulus differences (Terman, 1970; Blough, 1971). In the present context, the maximal level of stimulus control (i.e., few or no errors) could always be regained immediately by increasing the intensity to $25 \mathrm{~dB}$ from a smaller difference. For illustration, Fig. 1 presents on-line event recordings for both animals for 4-min segments before and after the transition from 1 to $25 \mathrm{~dB}$, representing approximately 100 trials at the end of a session at $p($ STD $)=$ 0.5 . The $1-\mathrm{dB}$ segments show near-chance performance, with the combined rate of false alarms and misses equalling approximately the combined rate of hits and correct rejections. Both error classes disappeared immediately when the stimulus difference was increased.

\section{RESULTS AND DISCUSSION} Discrimination Accuracy the reinforcer is obtained provides a convenient accuracy estimate (percent correct) in discrimination studies and has been used traditionally to generate the peychometric function. Figure 2 presents sample psychometric functions for Rat 7A in two standard probability conditions to illustrate the distortion in the accuracy estimate attributable to an O's response bias. The standard probability of 0.4 controlled nearly bias-free responding (equal proportion of "yes" and "no" errors), while 0.9 controlled a strong bias toward emitting "yes" errors (parametric data presentation to follow). Accordingly, the psychometric function for $p($ STD ) = 0.4 shows a steeper slope than 0.9 , with a cecuracy in the unbiased condition exceeding that in the biased condition for all stimulus differences less than $20 \mathrm{~dB}$. Both curves attain accuracy levels above $98 \%$ corrent at a 25-dB difference, indicating that the relative accuracy decrement is restricted to the transition region of the psychometric function. Such data suggest that the slope of the peychometric function and derived threshold estimates must be interpreted cautiously as sensory data in any experiment that has not verified bias-free performance.

\section{Isosensitivity Functions}

Signal detection theory suggests that while the percent correct measure is affected by both signal probability
The proportion of trials on which and intensity, the $d^{\prime}$ parameter may selectively reflect manipulations of signal intensity for all probabilities. Changes in probability determine the O's criterion for emitting the "yes" or "no" report, not the discriminability of the signal. Figure 3 (right panels) presents isosensitivity contours (from Elliott's table in Swets, 1964) for each animal, with the empirical data falling around them. The data agree with the curvilinear trend of the isosensitivity contours, with points on each curve falling closer to the major diagonal in the extreme bias conditions. Increasing signal intengity displaces the entire function away from the major diagonal, but the general trend of the function is maintained. From near-zero $d^{\prime} s$ at a 1 -dB difference, sensitivity increases for both animals to over $d^{\prime}=2$ at $10-d B$ difference. Such results recommend the use of $d^{i}$ as a valid index of auditory sensitivity for the rat. 1

\section{Difference Threshold}

(DL) Estimate

Given the general consistency of $d^{\prime}$ across isosensitivity functions, the change in sound pressure required to produce $d^{\prime}=1.0$ affords a convenient index of difference threshold for tone intensity (Engen, 1971). With the $100-\mathrm{dB}$ standard at $3.0 \mathrm{kHz}$, Rats $7 \mathrm{~A}$ and 15A showed DLs of 5.5 and 5.1 dB, respectively. Procedural variations make comparisons with DLs obtained in other experiments in animal psychophysics tentative, but certain comparisons may help to put these results in context. For example, Terman (1970) found a smaller DL of approximately $3.8 \mathrm{~dB}$ for a $4.0-\mathbf{k H z}$ sine tone at $100 \mathrm{~dB}$. In that experiment, tone durations greatly

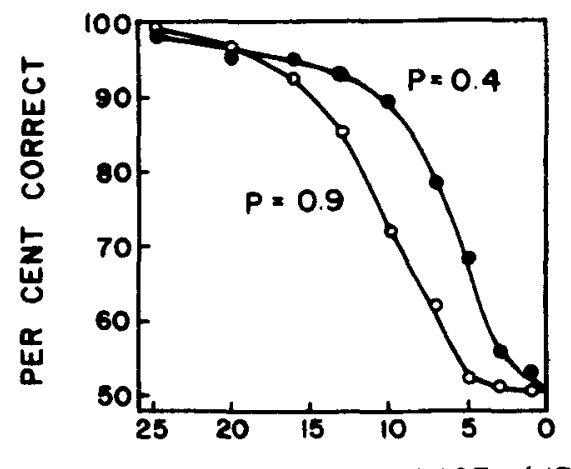

INTENSITY DIFFERENCE (dB)

Fig. 2. Paychometric functions for Rat $7 \mathrm{~A}$, under low- and high-bias conditions. Standard probability of 0.4 controlled nearly bias-free performance, while 0.9 controlled a strong bias toward emission of "yes" errors (false alarms). 
exceeded 0.5 sec, possibly increasing the discriminability of intensities (cf. Irwin \& Terman, 1970). Experiments in discrimination learning, using sine tone intensity as the controlling dimension (e.g.) Pierrel, Sherman, Blue, \& Hegge, 1970), have found a 5-dB attenuation from $100 \mathrm{~dB}$ at $4.0 \mathrm{kHz}$ to be an exceedingly difficult problem for the rat to master, with terminal accuracy never reaching near-errorless levels. The human $\mathrm{DL}$ is considerably finer than the rat's (Harris, 1963), but such a comparison is dubious, since the rat's best frequency exceeds man's upper limit by about an octave (Gourevitch \& Hack, 1966).

\section{Isobias Functions}

The left panels of Fig. 3 show successive points obtained as a function of decreasing the tone intensity difference for each standard probability condition. With decreasing intensity difference, each isobias function progresses from the upper-left corner of the unit square toward the major diagonal. The degree and direction of bias is reflected by the deviation of the curve from the minor diagonal (where the probability of false alarms and misges is equal, indicating zero bias). The loci of the isobias functions are distributed according to standard probability, with clear separation between adjacent functions. $p(S T D)=0.1$ controlled a strong bias toward "no" reports, while $p(S T D)=0.9$ controlled a strong bias toward "yes" reports. At intermediate probabilities, the loc of isobias functions are related monotonically to standard probability. It is important to note that at $p(S T D)=0.5$, the rats did not show zero bias but showed, instead, a moderate bias toward "yes" reports. For Rat 7A, unbiased performance is more closely approximated by the isobias function for $p$ (STD) $=0.4$. Rat 7A's isobias functions are fairly linear, describing a constancy in the proportion of false alarm to misses as the problem became more difficult (cf. Terman, 1970). Several of Rat 15A's functions bow out near the major diagonal suggesting a tendency toward maximizing either "yes" or "no" reports at small intensity differences, depending on the value of the standard probability (cf. Hodos, 1970).

Previous studies using two-choice p8ychophysical tests (Irwin \& Terman, 1970; Terman, 1970) have not obtained such clear control over bias. For example, Terman (1970) showed wide variability in the locus of isobias functions between animals which were all run at $p($ STD $)=0.5$. The development of position preferences during two-choice training may contribute to this result (see also
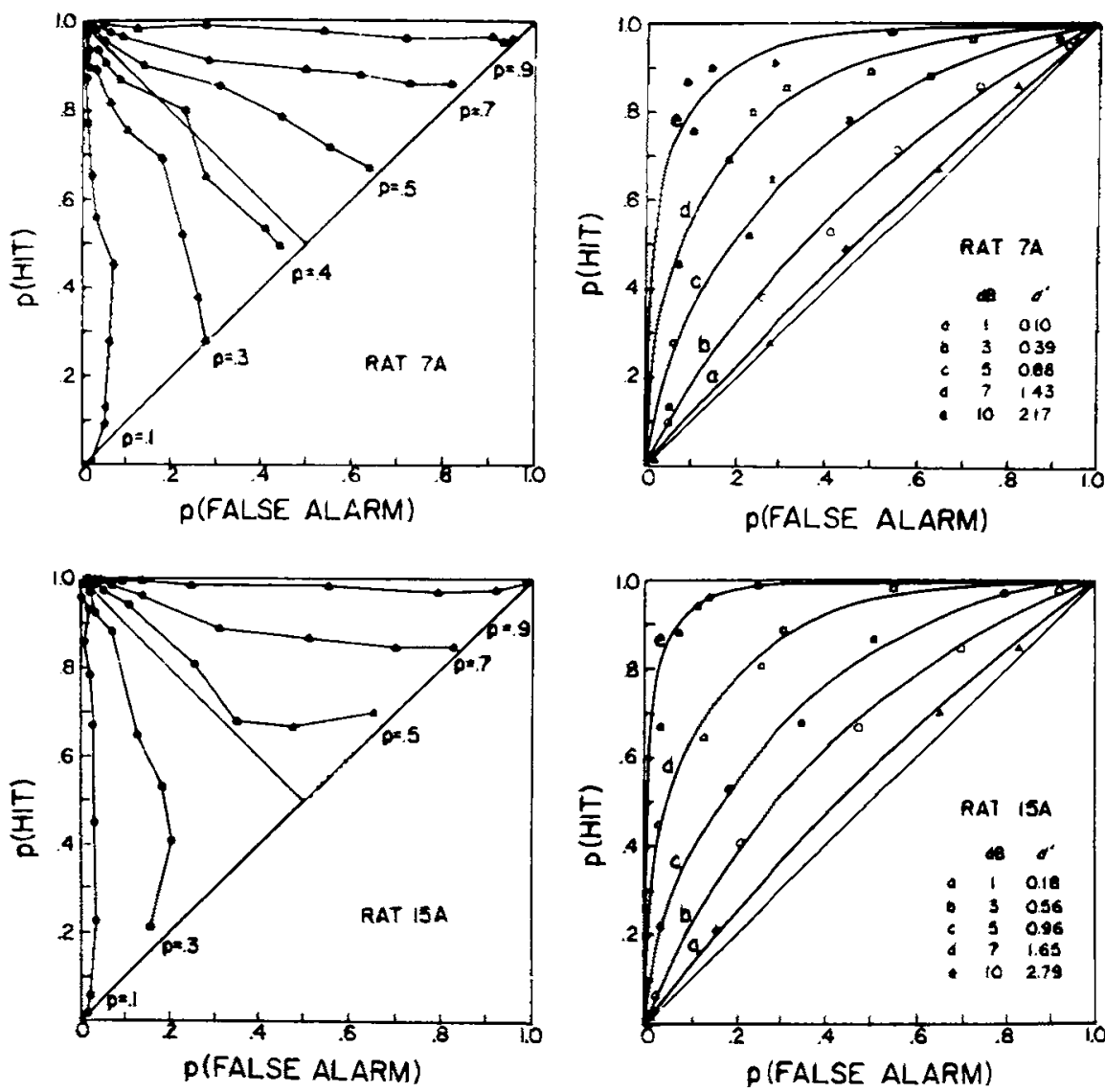

Fig. 3. Ioobias (left) and isosensitivity (right) functions for the sine-tone intensity discrimination tests. Probability values in the left-hand graphs indicate proportion of standard trials. $d^{\prime}$ contours in the right-hand graphs provide a theoretical estimate of sensitivity at various comparioon attenuation values.

Blough, 1971), for the distinctive right and left key-approach topographies that appear in two-choice experiments may determine response bias conjointly with signal probability (and/or payoff asymmetry). The single-key go/no-go design of the present experiment precludes the contribution of a position preference to the bias estimate and thus generates a more orderly analysis.

A peculiarity of the present single-key design is the dissimilarity of "yes" and "no" response definitions. A correct "no" report could be reinforced only after a delay of $3 \mathrm{sec}$ from comparison presentation, while a correct "yes" report was reinforced as soon as the keypress was emitted (usually after a delay of 0.7 to $1.2 \mathrm{sec})$. Thus, even though the payoff matrix was formally symmetrical, a distinct bias toward "yes" reports was obtained. For example, at p(STD) = 0.5 , the isobias function was displaced upward from the minor diagonal.

Probability Control at

Large Stimulus Differences

The relative contribution of the intensity and probability variables to the distribution of "yes" and "no" reports varies with discrimination difficulty. At large standardcomparison differences, the differential behavior is controlled by the auditory intensity dimension; nearly every standard presentation leads to a "yes" report (keypress), while nearly every comparison presentation leads to a "no" report Under these conditions, the overall frequency of "yes" and "no" reports closely matches the programmed standard probability, for the animal correctly identifies nearly every stimulus; thus, at large stimulus differences, we cannot attribute the overall proportion of "yes" and "no" reports to the probability variable. However, it would be a mistake to dismiss the potency of the probability variable per se at large stimulus differences. Both signal detection theory's treatment of overlapping normal distributions on the sensory continuum (Green \& Swets, 1966) and direct examination of the data show that some errors will always continue to occur regardless of the size of the 


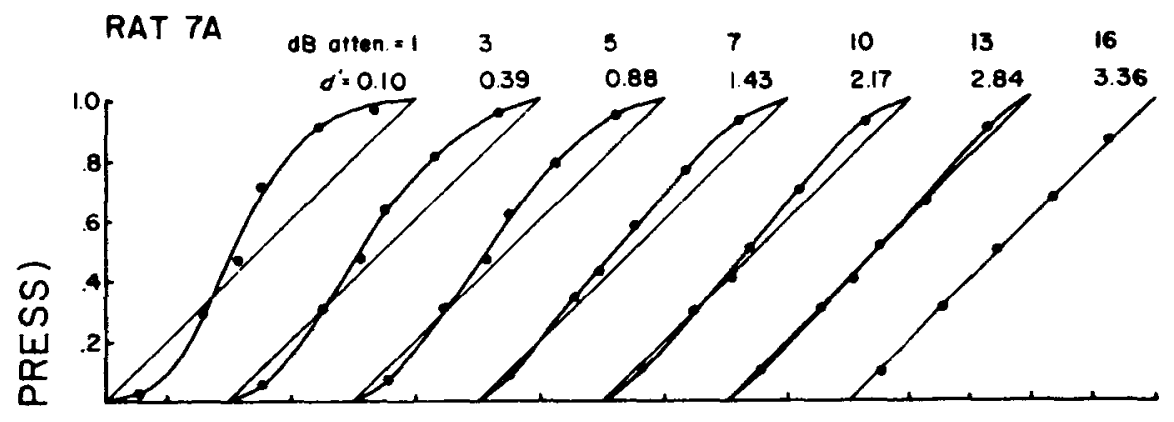

$>$ RAT I5A

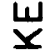

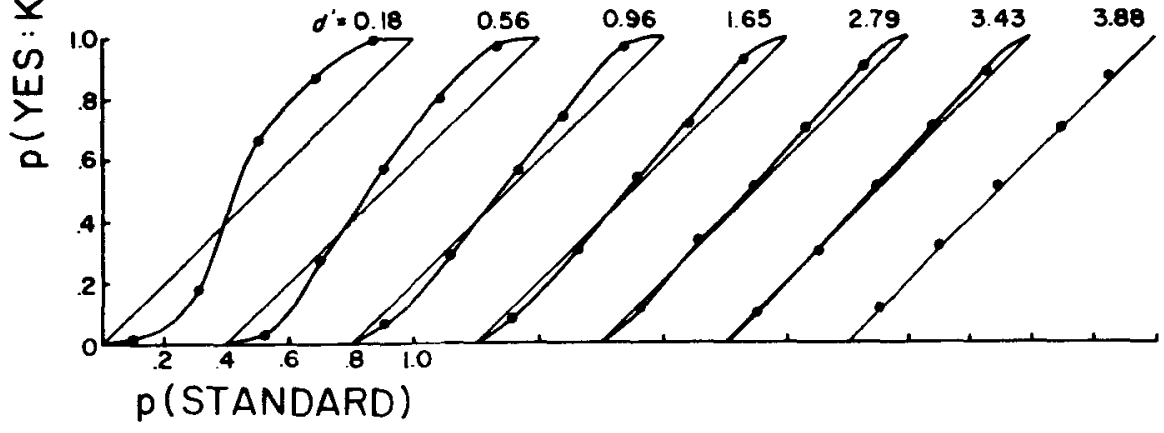

Fig. 4. Proportion of "yes" reports as a function of standard probability. Curves are displaced along the abscissa, with the corresponding straight-line function predicted by a matching rule. Sigmoid functions were fitted by eye.

stimulus intensity difference. At a 25-dB difference in this experiment, for example, errors typically occurred about once in 50 trials (yielding upper accuracy levels of about $98 \%$ correct). We can use these few occasions to show how the probability variable maintains control over the proportion of "yes" and "no" reports, since the error type (miss or false alarm probability, estimated over 1,400 trials) is clearly determined by the prevailing signal probability. Table 1 compares miss and false alarm probabilities at a $25-\mathrm{dB}$ stimulus difference for the two extreme probability conditions $(0.1$ and 0.9$)$. While all error rates were low, the error type corresponding to the predominant stimulus (misses at 0.1 , false alarms at 0.9 ) always exceeded the alternate error type. If the probability variable exerted no control at large stimulus differences, the error rates would be about equal.

Probability Control at

Small Stimulus Differences

The control by signal probability over the proportion of "yes" and "no" reports becomes ascendant at intermediate and small stimulus differences, for errors are emitted on a much greater proportion of trials. Indeed, at minimal intensity differences (e.g., $1 \mathrm{~dB}$ ), stimulus control is virtually absent and the animal has only the probability dimension to guide its choice between a "yes" or "no." Here the experimental paradigm converges on probability learning: the animal may maximize the number of reinforcers delivered only by emitting the most frequently reinforced response on every trial, since tone intensity no longer provides differential information. Alternatively, the animal may continue to emit "yes" and "no" reports in direct proportion to their frequency of reinforcement (a matching strategy), since such a behavioral distribution is reinforced at the larger intensity differences.

The data indicate that both animals matching strategy as the discrimination problem became more difficult. Figure 4 correlates the probability of "yes" reports with standard probability, for intensity differences ranging from 1 to $16 \mathrm{~dB}$. The linear diagonals represent the one-to-one correspondence of "yesses" to standards averaged over trials: the model for matching. As the

Table 1

Comparicon of Mins and False Alarm Probabilities at 25 dB Stimulus Difference

\begin{tabular}{cccccc} 
& \multicolumn{2}{c}{ Rat 7A } & & \multicolumn{2}{c}{ Rat 16A } \\
\cline { 2 - 3 } \cline { 5 - 6 } p(STD) & p(M) & p(FA) & & p(M) & p(FA) \\
\hline 1 & .130 & .009 & & .047 & .001 \\
.9 & .004 & .008 & .001 & .010 \\
\hline
\end{tabular}
deviated systematically from a strict by-product of high accuracy responding, the correspondence holds closely at the largent intencity difference plotted (16 dB, with $d^{\prime}$ above 3.30 for both animals). However, with decreaking intenxity differences, the frequency of "yes" reports exceeded that predicted by a matching rule at high probabilities (points above the diagonal), while at low probabilities there were fewer "yes" reports than predicted. The curve croms the matching line at a point below p(STD) $=0.5$, demonstrating the paychological asymmetry of the "yes" (keypreas) and "no" (wait for 3 sec) reports. Interestingly, the animals did not maximize acroes all probability values [yielding a step function from $p(y e s)=$ 0 to 1.0 ] at the nearly imponsible discriminations of 1 and $3 \mathrm{~dB}$, although point at the extreme values $(0.1$ and 0.9$)$ were close to maximizing. Under these low-accuracy conditions, both animals showed a sigmoid function relating "yes" reports to standard probability.

In summary, it may be said that, while matching is an inevitable by-product of high-accuracy responding at large stimulus differences, the proportion of "yes" and "no" responses may deviate considerably from a matching rule during difficult discriminations, with a resulting gain in total reinforcements. In fact, matching and maximizing represent two behavioral extremes on the continuum of yes-no response proportions, and the interaction between stimulus intensity and probability variables in a signal detection test may lead to a unique behavioral function that cannot be simply described as either a product of probability learning or stimulus control in vacuo. Perhaps when a psychophysical test session has started at wide stimulus differences, control by the auditory stimulus dimension lingers for a period, even after its differential function has been eliminated or reduced. If so, we would expect extended sessions at minimal stimulus difference eventually to support maximizing. The time course over which the matching strategy transforms to a maximizing strategy (cf. Shimp, 1966) may provide an index of the loes of control by the psychophysical stimulus dimension in difficult discriminations, well a more comprehensive account of the shape of isobias functions.

\section{REFERENCES}

BERKLEY, M. A. \& KIING, J. W. A small animal coupler with vertical movement compensations. Physiolory \& Behavior. 1867, 2, 316-316.

BLOUGH, D. S. Stimulue generalization as sirnal detection in pireons. Seience. $1967,158,940-941$. 
BLOUGH, P. M. The visual acuity of the pigeon for distant targets. Journal of the Experimental Analysis of Behavior, 1971, 15, 57-67.

ENGEN,T. Psychophysics: I Discrimination and detection. In $J$. $W$ Kling and L. A. Riggs (Eds.), Woodworth and Schlosberg's experimental psychology. (3rd ed.) New York: Holt, Rinehart \& winston, 1971 .

GOUREVITCH, G., \& HACK, M. H. A udibility in the rat. Journal of Comparative \& Physiological Psychology, 1966, 62, 289-291.

GREEN, D. M., \& SWETS, J. A. Signal detection theory and psychophysica New York: Wiley, 1966.

HARRIS, J. D. Loudness discrimination. Journal of Speech \& Hearing Disorders, Monograph Suppl. II, 1963.

HODOS, W. A non-parametric index of response bias for use in detection and recognition experiments. Psychological Bulletin, 1970, 74, 351-354.

IR WIN, R. J., \& TERMAN, M. Detection of brief tones in noise by rats. Journal of the Experimental Analysis of Behavior, 1970 , $13,135-143$.

KELLER, F. S., \& SCHOENFELD, W. N Principles of psychology. New York: Appleton-Century-Crofts, 1950.

NEVIN, J. A. On differential stimulation and differential reinforcement. In $W$. C Stebbins (Ed.), Animal psychophysics. New York: Appleton-Century-Crofts, 1970.

PIERREL, R., SHERMAN, J. G., BLUE, S. \& HEGGE, F. W. Auditory discrimination: A three-variable analysis of intensity effects. Journal of the Experimental Analysis of Behavior, 1970 13, 17-35.
SHIMP, C. P. Probabilistically reinforeed choice behavior in pigeons. Journal of the Experimental Analysis of Behavior, 1966, $9,443-455$.

SWETS, J. A. Signal detection and recognition by human observers. New York: Wiley, 1964.

TERMAN, M. Discrimination of auditory intensities by rats. Journal of the Experimental Analysis of Behavior, 1970 , $13,145-160$.

NOTE

1. Rat 7A's reduced $d^{\prime}$ values at $p(S T D)=$ 0.1 (leftmost points in Fig. 3) reflect an excessive miss rate even at the largest intensity difference. In future experiments, adjustment of reinforcer parameters might minimize this problem.

(Received for publication July 21, 1971.) 